\title{
CR-CONTINUATION OF ARC-ANALYTIC MAPS
}

\author{
JANUSZ ADAMUS
}

(Communicated by Franc Forstneric)

\begin{abstract}
Given a set $E$ in $\mathbb{C}^{m}$ and a point $p \in E$, there is a unique smallest complex-analytic germ $X_{p}$ containing $E_{p}$, called the holomorphic closure of $E_{p}$. We study the holomorphic closure of semialgebraic arc-symmetric sets. Our main application concerns CR-continuation of semialgebraic arc-analytic mappings: A mapping $f: M \rightarrow \mathbb{C}^{n}$ on a connected real-analytic $\mathrm{CR}$ manifold which is semialgebraic arc-analytic and CR on a non-empty open subset of $M$ is $\mathrm{CR}$ on the whole $M$.
\end{abstract}

\section{INTRODUCTION}

Let $\Omega$ be an open set in $\mathbb{R}^{m}$. A function $f$ real-analytic in $\Omega$ is called Nash if there is a non-constant polynomial $P \in \mathbb{R}[x, y]$, where $x=\left(x_{1}, \ldots, x_{m}\right)$, such that $P(x, f(x))=0$ for all $x \in \Omega$. A real-analytic set is a Nash set if it is (locally) defined by Nash real-analytic functions.

In the present note we will be concerned with a more general class of functions which appear naturally in real geometry. Given a real-analytic set $R$ in an open $\Omega \subset \mathbb{R}^{m}$, a function $f: R \rightarrow \mathbb{R}$ is called arc-analytic if it is analytic on every arc, that is, if $f \circ \gamma$ is analytic for every real-analytic $\gamma:(-\varepsilon, \varepsilon) \rightarrow R$.

Recall that a set $E$ in $\mathbb{R}^{m}$ is called semialgebraic if it is a finite union of sets of the form

$$
\left\{x=\left(x_{1}, \ldots, x_{m}\right) \in \mathbb{R}^{m}: f_{1}(x)=\cdots=f_{r}(x)=0, g_{1}(x)>0, \ldots, g_{s}(x)>0\right\},
$$

where $r, s \in \mathbb{N}$ and $f_{1}, \ldots, f_{r}, g_{1}, \ldots, g_{s} \in \mathbb{R}[x]$. A function $f: E \rightarrow \mathbb{R}$ is called semialgebraic if its graph $\Gamma_{f}$ is a semialgebraic subset of $\mathbb{R}^{m} \times \mathbb{R}$. A semialgebraic mapping $f=\left(f_{1}, \ldots, f_{n}\right): E \rightarrow \mathbb{R}^{n}$ is one in which all of its components $f_{j}$ are semialgebraic. Identifying $\mathbb{C}^{m}$ with $\mathbb{R}^{2 m}$, one can speak of real-analytic, Nash, and semialgebraic subsets of $\mathbb{C}^{m}$.

Our main result is the following variant of a theorem of Shafikov [14] on CRcontinuation of continuous mappings (for details on CR structure and CR functions, see Section 2).

Theorem 1.1. Let $M$ be a connected Nash real-analytic CR submanifold of an open set in $\mathbb{C}^{m}$, and let $f: M \rightarrow \mathbb{C}^{n}$ be a semialgebraic arc-analytic mapping $(m, n \geq 1)$. If $f$ is $C R$ on a nonempty open subset of $M$, then $f$ is $C R$ on $M$.

Received by the editors January 18, 2014 and, in revised form, June 3, 2014.

2010 Mathematics Subject Classification. Primary 14P20, 32V10; Secondary 14P10, 32V40, $32 \mathrm{~V} 20$.

Research was partially supported by Natural Sciences and Engineering Research Council of Canada. 
Semialgebraic arc-analytic mappings are necessarily continuous [9, Proposition 5.1]. However, an arc-analytic map need not have a real-analytic graph (see Example 1.2 below). It follows that Theorem 1.1 is strictly stronger than Shafikov's [14, Theorem 1.3] in the Nash setting.

Note also that semialgebraicity itself would not suffice to obtain the conclusion of Theorem 1.1. Indeed, a continuous semialgebraic map $f: M \rightarrow \mathbb{C}^{n}$ may have very different $\mathrm{CR}$ properties on different open subsets of $M$. Consider, for instance, $M=\mathbb{C}$ and a function $f: M \rightarrow \mathbb{C}$ defined as $f(x+i y)=\sqrt{x^{4}+y^{4}}$ for $x^{4}+y^{4} \leq 1$ and $f(x+i y)=1$ for $x^{4}+y^{4} \geq 1$. Then $f$ is holomorphic in some open neighbourhood of every $z_{0}$ with, say, $\left|z_{0}\right|>2$, but $f$ is not CR on $M$.

Arc-analytic functions, although relatively unknown among non-specialists, form a very important class in real-analytic geometry. Indeed, Bierstone and Milman proved in [6] that arc-analytic semialgebraic functions on a Nash manifold are precisely those that can be made Nash analytic after composition with a finite sequence of blowings-up with smooth nowhere-dense centers. Many classical examples in calculus are arc-analytic but not analytic.

Example 1.2. (a) The function $f: \mathbb{R}^{2} \rightarrow \mathbb{R}$ defined as $f(x, y)=x^{3} /\left(x^{2}+y^{2}\right)$ for $(x, y) \neq(0,0)$ and $f(0,0)=0$ is arc-analytic but not differentiable at the origin. (Observe that $f$ is made Nash after composition with a single blowing-up of the origin; for instance, $f(x, x y)=x /\left(1+y^{2}\right)$.) Note also that the graph $\Gamma_{f}$ of $f$ is not real-analytic. In fact, the smallest real-analytic subset of $\mathbb{R}^{3}$ containing $\Gamma_{f}$ is the Cartan umbrella $\left\{(x, y, z) \in \mathbb{R}^{3}: z\left(x^{2}+y^{2}\right)=x^{3}\right\}$ (cf. [9, Example 1.2(1)]).

(b) The function $g: \mathbb{R}^{2} \rightarrow \mathbb{R}$ defined as $g(x, y)=\sqrt{x^{4}+y^{4}}$ is arc-analytic but not $\mathcal{C}^{2}$. The graph $\Gamma_{g}$ of $g$ is not real-analytic. Indeed, the Zariski closure $\left\{(x, y, z) \in \mathbb{R}^{3}: z^{2}=x^{4}+y^{4}\right\}$ of $\Gamma_{g}$ has two $\mathcal{C}^{1}$ sheets $z= \pm \sqrt{x^{4}+y^{4}}$, but it is irreducible at the origin as a real-analytic set (cf. [6. Example 1.2(3)]).

The main tool used in the proof of Theorem 1.1 is the so-called holomorphic closure (HC for short). Given a set $E$ in $\mathbb{C}^{m}$ and a point $p \in E$, one defines the holomorphic closure of the germ $E_{p}$ as the unique smallest (with respect to inclusion) complex-analytic germ which contains $E_{p}$; it is denoted ${\overline{E_{p}}}^{H C}$. The holomorphic closure dimension of $E_{p}, \operatorname{dim}_{H C} E_{p}$, is the (complex) dimension of ${\overline{E_{p}}}^{H C}$. Holomorphic closure of real-analytic germs in complex spaces had been studied in [14], 4], and [2. It is closely connected with the CR structure (see, e.g., [4, Proposition 1.4 and Theorem 1.5]). In [2] we considered holomorphic closure in the semialgebraic category. We showed there that the $\mathrm{HC}$ dimension is tame in this category, which was used to prove the existence of semialgebraic stratification by CR manifolds.

In the present paper we continue the study of the $\mathrm{HC}$ structure of semialgebraic sets. This time we investigate how the $\mathrm{HC}$ dimension behaves on arc-symmetric sets. Recall that a set $E \subset \mathbb{R}^{m}$ is called arc-symmetric when, for every real-analytic arc $\gamma:(-1,1) \rightarrow \mathbb{R}^{m}$ with $\gamma((-1,0)) \subset E$, there exists $\varepsilon>0$ such that $\gamma((-1, \varepsilon)) \subset E$ (cf. [9, Definition 1.1]). The concept of arc-symmetry was introduced by Kurdyka [9] in the semialgebraic category. It allows one to make sense of the notions of irreducibility and components of a semialgebraic set much like in the algebraic case (see Section 2 for details).

Semialgebraic arc-symmetric subsets of $\mathbb{R}^{m}$ will be called $\mathcal{A R}$-closed sets (cf. Theorem 2.5). The following result lies at the center of our arguments. 
Theorem 1.3. Let $E \subset \mathbb{C}^{m}$ be a semialgebraic $\mathcal{A R}$-irreducible set of pure dimension. Then

(i) There exists an integer $h$ such that $\operatorname{dim}_{H C} E_{p}=h$ for all $p \in E$.

(ii) If $Z$ is the smallest complex-algebraic set in $\mathbb{C}^{m}$ containing $E$, then $Z$ is irreducible and of (complex) dimension $h$.

Note that the set $Z$ in the above theorem need not realize the HC closure of $E$ at each of its points (see, e.g., [14, §2] or [1, Example 4.4]). It does so, however, when $E$ has a complex-analytic germ at some point. The following result is an arc-symmetric analogue of Shafikov's [14, Cor. 1.2]. It will be used in the proof of Theorem 1.1

Theorem 1.4. Let $E \subset \mathbb{C}^{m}$ be a semialgebraic $\mathcal{A R}$-irreducible set of pure dimension. If $E$ contains a point $p$ such that $E_{p}$ is a complex-analytic germ, then $E$ is irreducible complex-algebraic.

In the next section we recall basic definitions and tools used in this article. Theorems 1.3 and 1.4 are proved in Section 3, which is devoted to the study of holomorphic closure of arc-symmetric sets. The last section contains the proof of Theorem 1.1.

\section{Preliminaries}

2.1. CR manifolds and CR functions. Given an $\mathbb{R}$-linear subspace $L$ in $\mathbb{C}^{m}$ of dimension $d$, one defines the $C R$ dimension of $L$ to be the largest $k$ such that $L$ contains a $\mathbb{C}$-linear subspace of (complex) dimension $k$. Clearly, $0 \leq k \leq\left[\frac{d}{2}\right]$. A $d$-dimensional real-analytic submanifold $M$ of an open set in $\mathbb{C}^{m}$ is called a $C R$ manifold of $\mathrm{CR}$ dimension $k$, if the tangent space $T_{p} M$ has $\mathrm{CR}$ dimension $k$ for every point $p \in M$ (the $k$-dimensional complex vector subspace of $T_{p} M$ will then be denoted by $\left.H_{p} M\right)$. We write $\operatorname{dim}_{C R} M=k$. If $k=0$, then $M$ is called a totally real submanifold. The integer $l:=d-2 k$ is called the $C R$ codimension of $M$, and the pair $(k, l)$ is the type of $M$. A CR submanifold $M$ in $\mathbb{C}^{m}$ is called generic when $m=k+l$, where $M$ is of type $(k, l)$.

The notion of $C R$ function is usually defined in terms of tangential CauchyRiemann equations, as follows. Given a real-analytic CR submanifold $M$ in an open set in $\mathbb{C}^{m}$, a smooth vector field $X$ on $M$ is called a $C R$ vector field if $X_{p} \in H_{p} M$ for every $p \in M$. A $\mathcal{C}^{1}$-smooth function $f: M \rightarrow \mathbb{C}$ is $\mathrm{CR}$ if $X f \equiv 0$ for every $\mathrm{CR}$ vector field $X$ on $M$ of the form $X=\sum_{j=1}^{m} c_{j} \frac{\partial}{\partial \bar{z}_{j}}$. However, in order to use Theorem 1.1 in its full generality, we shall need a more general definition that does not require smoothness of $f$. This can be done in terms of distributions: Suppose that $M$ is of type $(k, l)$. A locally integrable function $f: M \rightarrow \mathbb{C}$ is called a $C R$ function if

$$
\int_{M} f \bar{\partial} \alpha=0
$$

for any differential form $\alpha$ of bidegree $(k, k+l-1)$ with compact support (cf. [13, §21]). A CR mapping $f=\left(f_{1}, \ldots, f_{n}\right): M \rightarrow \mathbb{C}^{n}$ is one in which all of its components $f_{j}$ are CR functions.

2.2. Holomorphic closure of a semialgebraic set germ. Given a set $E$ in $\mathbb{C}^{m}$ and a point $p \in E$, one defines the holomorphic closure of the germ $E_{p}$ as the unique smallest (with respect to inclusion) complex analytic germ which contains 
$E_{p}$; it is denoted ${\overline{E_{p}}}^{H C}$. The holomorphic closure dimension of $E_{p}, \operatorname{dim}_{H C} E_{p}$, is the (complex) dimension of ${\overline{E_{p}}}^{H C}$. For $d \in \mathbb{N}$, we denote by $\mathcal{S}^{d}(E)$ the set of those points $p \in E$ for which $\operatorname{dim}_{H C} E_{p} \geq d$.

For the reader's convenience, we recall the following results from [4] and [2] that will be used in the present paper.

Theorem 2.1 (cf. 4, Theorem 1.3]). Let $E \subset \mathbb{C}^{m}$ be a connected semialgebraic set of pure dimension, contained in an irreducible real-analytic set of the same dimension. Then the holomorphic closure dimension is constant on E.

Lemma 2.2 ([2, Lemma 2]). Let $E$ be a connected real-analytic submanifold of an open set in $\mathbb{C}^{m}$. There exists a unique smallest complex-algebraic subset $Z$ of $\mathbb{C}^{m}$ containing $\bar{E}$ and such that, for every $p \in \bar{E}, Z_{p}$ is the smallest complex-algebraic germ containing $E_{p}$. Moreover, $Z$ is irreducible.

Proposition 2.3 ([2, Proposition 5]). Let E be a connected semialgebraic realanalytic submanifold of an open subset of $\mathbb{C}^{m}$, and let $Z$ be the unique irreducible complex-algebraic set from the above lemma. Then, at every point $p \in \bar{E}$, the holomorphic closure ${\overline{E_{p}}}^{H C}$ is a union of some analytic-irreducible components of $Z_{p}$. In particular, the holomorphic closure dimension is constant on $E$.

2.3. Nash real-analytic sets. There is a little ambiguity in literature regarding (real) Nash sets. In fact, there are two common definitions, which do not coincide in general. According to one definition, a Nash set is a real-analytic subset $R$ of an open set $\Omega$ in $\mathbb{R}^{m}$ with the property that for every $p \in \Omega$ there is an open neighbourhood $U$ of $p$ in $\Omega$ such that $R \cap U$ is defined by the vanishing of finitely many functions that are Nash analytic in $U$ (as in Section 11). This is how realanalytic Nash sets are understood; for instance, in [5]. Another classical monograph [7] defines $R \subset \mathbb{R}^{m}$ to be Nash when it is real-analytic in an open $\Omega$ in $\mathbb{R}^{m}$ and a semialgebraic subset of $\mathbb{R}^{m}$.

Remark 2.4. It is not difficult to see that a set $R$ which is Nash in the second sense is also Nash in the first sense. The two notions coincide when $R$ is compact or, more generally, when $R$ admits a finite partition into connected smooth manifolds. (They also always coincide in the complex-analytic case.) However, in general, a set which is Nash in the first sense need not be semialgebraic (for example, its regular locus may have an infinite number of connected components).

As far as Theorem 1.1 goes, one can disregard the above ambiguity because the domain of a semialgebraic mapping $f$ is always semialgebraic (by the TarskiSeidenberg Theorem (see, e.g., [7, Proposition 2.2.7]) as a projection of the semialgebraic set $\left.\Gamma_{f}\right)$.

2.4. Semialgebraic arc-symmetric sets. The notion of arc-symmetry was introduced by Kurdyka 9] in the semialgebraic category. Its usefulness comes from the following fundamental result.

Theorem 2.5 ([9, Theorem 1.4]). There exists a unique noetherian topology $\mathcal{A R}$ on $\mathbb{R}^{m}$, such that the $\mathcal{A} \mathcal{R}$-closed sets are precisely the semialgebraic arc-symmetric sets in $\mathbb{R}^{m}$.

(A topology is called noetherian, when every descending sequence of closed sets is stationary.) For a detailed exposition of arc-symmetric sets, we refer the reader 
to [9] or [10. Here we recall only a few basic properties that will be used in the present paper.

The class of $\mathcal{A R}$-closed sets includes, in particular, the real-algebraic sets as well as the Nash real-analytic sets (in the sense of [7]). The $\mathcal{A R}$-topology is strictly finer than the Zariski topology on $\mathbb{R}^{m}$ (see, e.g., [9, Example 1.2]). Moreover, it follows from the semialgebraic Curve Selection Lemma that $\mathcal{A R}$-closed sets are closed in the Euclidean topology in $\mathbb{R}^{m}$ (see [9, Remark 1.3]).

An $\mathcal{A R}$-closed set $E$ is called $\mathcal{A R}$-irreducible, when $E=E_{1} \cup E_{2}$ with $E_{1}, E_{2}$ $\mathcal{A R}$-closed implies that $E=E_{1}$ or $E=E_{2}$. It follows from Theorem 2.5 that every $\mathcal{A R}$-closed set $E$ admits a (unique) finite decomposition $E=E_{1} \cup \cdots \cup E_{s}$ into $\mathcal{A R}$-irreducible subsets such that $E_{j} \not \subset \bigcup_{k \neq j} E_{k}$ for $j=1, \ldots, s$. The sets $E_{j}$ of this decomposition are called the $\mathcal{A R}$-irreducible components of $E$.

In the next section, we will study the HC structure of $\mathcal{A} \mathcal{R}$-closed sets in a complex space. These sets will play an important role in the proof of Theorem 1.1, because, according to [9, Proposition 5.1], the graph of a semialgebraic arc-analytic mapping is an $\mathcal{A R}$-closed set.

\section{HolOMORPHIC ClOSURE OF AN ARC-SYMMETRIC SET}

In general, $\mathcal{A R}$-closed sets (even $\mathcal{A R}$-irreducible) need not be of pure holomorphic closure dimension. Even worse than that, the HC-filtration $\left\{\mathcal{S}^{d}(E)\right\}_{d \in \mathbb{N}}$ of an $\mathcal{A R}$-closed set $E$ need not be $\mathcal{A R}$-closed itself. For example, the Whitney umbrella embedded in $\mathbb{C}^{2}$ as

$$
V=\left\{\left(x_{1}+i y_{1}, x_{2}+i y_{2}\right) \in \mathbb{C}^{2}: x_{1}^{2}-x_{2} y_{1}^{2}=y_{2}=0\right\}
$$

happens to be $\mathcal{A R}$-irreducible (cf. [9, Remark 3.2]), but $\mathcal{S}^{2}(V)$ coincides with the two-dimensional part of $V$ (since the "stick" $\left\{x_{1}=y_{1}=y_{2}=0\right\}$ is contained in a complex line), which is not $\mathcal{A R}$-closed.

Nonetheless, according to Theorem 1.3 the above problems can occur only in the mixed-dimensional case.

3.1. Proof of Theorem 1.3. The proof of Theorem 1.3 will rely on the following two lemmas.

Lemma 3.1. Let $E$ be an $\mathcal{A R}$-irreducible set in $\mathbb{C}^{m}$. If $Z$ is the smallest (with respect to inclusion) complex-algebraic set containing $E$, then $Z$ is irreducible.

Proof. Suppose that $Z=Z_{1} \cup Z_{2}$ is a union of two complex-algebraic sets, with $Z_{j} \neq Z, j=1,2$. The sets $E \cap Z_{1}, E \cap Z_{2}$ being semialgebraic and arc-symmetric, it follows that $E \subset Z_{1}$ or $E \subset Z_{2}$ (by $\mathcal{A R}$-irreducibility of $E$ ). This contradicts the minimality of $Z$.

Given a real-analytic or semialgebraic set $E$ and $d \in \mathbb{N}$, we denote by $\operatorname{Reg}_{d} E$ the locus of points $p \in E$ such that $E_{p}$ is a germ of a $d$-dimensional manifold.

Lemma 3.2. Let $E$ be a $k$-dimensional $\mathcal{A R}$-irreducible set in $\mathbb{C}^{m}$. If $R$ is the smallest real-analytic set containing $\overline{\operatorname{Reg}_{k}(E)}$, then $R$ is irreducible and of dimension $k$. 
Proof. Observe first that $R$ is well defined, since the intersection of the family of all real-analytic sets in $\mathbb{C}^{m}$ containing $\overline{\operatorname{Reg}_{k}(E)}$ is itself real-analytic (see, e.g., [12, Ch. V, $\S 2$, Cor. 2]). Since the Zariski closure of the semialgebraic set $\overline{\operatorname{Reg}_{k}(E)}$ (i.e., the smallest real-algebraic set containing $\overline{\operatorname{Reg}_{k}(E)}$ ) is $k$-dimensional (see [7, $\left.\S 2.8\right]$ ), it follows that $\operatorname{dim} R=k$.

Suppose then that $R=R_{1} \cup R_{2}$ is a union of two real-analytic sets, with $R_{j} \neq R$, $j=1,2$. Since $\operatorname{Reg}_{k}(E) \subset R_{1} \cup R_{2}$, either $R_{1}$ or $R_{2}$ must contain a non-empty open subset of $\operatorname{Reg}_{k}(E)$; say, the former. Let $\operatorname{Reg}_{k}(E)=E_{1} \cup \cdots \cup E_{s}$ be the decomposition into (finitely many, by semialgebraic stratification) connected components. After renumbering the components if needed, we get that $R_{1}$ contains a non-empty open subset of $E_{1}$, and hence $E_{1} \subset R_{1}$, by the Identity Principle. By closedness of $R_{1}$, we get $\bar{E}_{1} \subset R_{1}$.

Now, after renumbering the components if needed, we can assume that $\bar{E}_{1}, \ldots$, $\bar{E}_{t} \subset R_{1}$ and $\bar{E}_{t+1}, \ldots, \bar{E}_{s} \not \subset R_{1}$, for some $t \leq s$. If $t=s$, then $\overline{\operatorname{Reg}_{k}(E)}=$ $\bar{E}_{1} \cup \cdots \cup \bar{E}_{s} \subset R_{1}$, which contradicts the choice of $R$. Hence $t<s$, and so $E_{s} \not \subset R_{1}$. Choose arbitrary $p \in E_{s} \backslash R_{1}$ and $q \in E_{1}$. By $\mathcal{A} \mathcal{R}$-irreducibility of $E$ and [9. Cor. 2.8], there is an arc $\gamma:[0,1] \rightarrow \overline{\operatorname{Reg}_{k}(E)}$ analytic in a neighbourhood of $[0,1]$ and such that $\gamma(0)=q$ and $\gamma(1)=p$. Since $q \in E_{1}$, we have $\operatorname{Int}\left(\gamma^{-1}\left(R_{1}\right)\right) \neq \varnothing$. But $R_{1}$ is arc-symmetric (as a real-analytic set), and so $\gamma([0,1]) \subset R_{1}$. In particular, $p \in R_{1}$; a contradiction. This shows that $R$ is irreducible.

Proof of Theorem 1.3. If $E$ is of pure dimension $k$, then $E=\overline{\operatorname{Reg}_{k}(E)}$. Hence, by Lemma 3.2, the smallest real-analytic set $R$ containing $E$ is irreducible and of dimension $k$. The claim (i) thus follows from Theorem 2.1.

For the proof of (ii), let $\operatorname{Reg}_{k}(E)=E_{1} \cup \cdots \cup E_{s}$ be the decomposition into connected components. By Proposition 2.3, for every $j$, there is a unique smallest complex-algebraic set $Z_{j}$ such that ${\overline{\left(E_{j}\right)_{p}}}^{H C}$ is a union of certain analytic-irreducible components of $\left(Z_{j}\right)_{p}$ for all $p \in E_{j}$. By part (i) of Theorem 1.3. $\operatorname{dim} Z_{j}=h$ for $j=1, \ldots, s$, and so $E=\overline{\operatorname{Reg}_{k}(E)}$ is contained in an $h$-dimensional complexalgebraic set $Z_{1} \cup \cdots \cup Z_{s}$. Now, let $Z$ be the smallest complex-algebraic set that contains $E$. By Lemma 3.1, $Z$ is irreducible. Hence, $Z$ is of pure dimension and, clearly, $\operatorname{dim} Z \leq \operatorname{dim}\left(Z_{1} \cup \cdots \cup Z_{s}\right)=h$. On the other hand, $\operatorname{dim} Z \geq h$, for otherwise the constant $\mathrm{HC}$ dimension of $E$ would be less than $h$.

3.2. Corollaries of Theorem 1.3. We will now derive two simple consequences of Theorem 1.3 .

Proof of Theorem 1.4. Fix $p \in E$ and a semialgebraic open neighbourhood $U$ of $p$ in $\mathbb{C}^{m}$ such that $E \cap U$ is a complex-analytic subset of $U$. Then $E \cap U$ is a complex Nash subset of $U$, by [2, Proposition 3]. Let $X$ be an analytic irreducible component of $E \cap U$ of maximal dimension. By [15, Theorem 2.10], there is an irreducible complex-algebraic set $Y$ in $\mathbb{C}^{m}$ such that $X$ is an irreducible component of $Y \cap U$.

Let $Z$ be the unique smallest complex-algebraic set containing $E$. Set $k:=\operatorname{dim} E$. Since $E \cap U$ is complex-analytic, we get that $\operatorname{dim}_{\mathbb{C}} X_{p}=\operatorname{dim}_{H C} E_{p}=\operatorname{dim}_{\mathbb{R}} E_{p} / 2$. Hence $X$ is of pure dimension $h:=k / 2$, and $h$ is the constant holomorphic closure dimension of $E$. By Theorem 1.3(ii), $\operatorname{dim} Z=h$. By irreducibility of $Y$, we also have that $\operatorname{dim} Y=\operatorname{dim} X=h$. Since $X \subset Z \cap Y$, we have $\operatorname{dim}(Z \cap Y) \geq \operatorname{dim} X=h$, and hence $Z=Y$, by irreducibility. 
It follows that $E$ contains a non-empty open subset of $Z$, and $\operatorname{so} \operatorname{dim}(\operatorname{Reg} Z \cap E)=$ $\operatorname{dim} \operatorname{Reg} Z$. By irreducibility of $Z, \operatorname{Reg} Z$ is a connected (semialgebraic) smooth manifold (cf. [11, Ch. VII, $\S 11.1]$ ), and thus $\operatorname{Reg} Z \subset E$, by [9, Remark 1.6]. Therefore, $Z=\overline{\operatorname{Reg} Z} \subset E \subset Z$, so $E=Z$ is irreducible complex-algebraic, as required.

Another consequence of Theorem 1.3 is the following observation (cf. example at the beginning of this section).

Corollary 3.3. Let $E$ be an arc-symmetric set in $\mathbb{C}^{m}$. If $E$ is of pure dimension, then its $H C$ filtration is $\mathcal{A R}$-closed, that is, $\mathcal{S}^{d}(E)$ is $\mathcal{A R}$-closed for every $d \in \mathbb{N}$.

Proof. Let $E=E_{1} \cup \cdots \cup E_{s}$ be the decomposition of $E$ into $\mathcal{A R}$-irreducible components. Then each $E_{j}$ is of pure dimension and, hence, of pure HC dimension by Theorem 1.3. It follows that, for a given $d \in \mathbb{N}, \mathcal{S}^{d}(E)$ is the union of those $E_{j}$ whose $\mathrm{HC}$ dimension is at least $d$. Therefore $\mathcal{S}^{d}(E)$ is $\mathcal{A} \mathcal{R}$-closed as a finite union of $\mathcal{A R}$-closed sets.

Remark 3.4. The above corollary has no analogue for the (inner) complex dimension. That is, a pure-dimensional semialgebraic arc-symmetric set need not have $\mathcal{A R}$-closed filtration by the complex dimension. We will use the following notation from [3]: For a real analytic set $R$ in $\mathbb{C}^{m}$ and $d \in \mathbb{N}$, let $\mathcal{A}^{d}(R)$ denote the set of points $p \in R$ such that $R_{p}$ contains a complex-analytic germ of (complex) dimension $d$. Now, the irreducible real-algebraic hypersurface

$$
X=\left\{\left(z_{1}, \ldots, z_{4}\right) \in \mathbb{C}^{4}: x_{1}^{2}-x_{2}^{2}+x_{3}^{2}=x_{4}^{3}\right\},
$$

where $z_{j}=x_{j}+i y_{j}$, satisfies $\mathcal{A}^{1}(X)=X \cap\left\{x_{4} \geq 0\right\}$ near the point $p=(1,1,0,0)$. (We know from [3, Theorem 1.1] that $\mathcal{A}^{1}(X)$ is a closed semialgebraic subset of $X$.) But $X$ is a 7 -manifold near $p$ (in fact, everywhere except at the origin); hence, $\mathcal{A}^{1}(X)$ contains a non-empty open subset of $\operatorname{Reg}_{7} X=X \backslash\{0\}$. By Kurdyka's [9. Remark 1.6], if $\mathcal{A}^{1}(X)$ were arc-symmetric, it would need to contain the whole $X \backslash\{0\}$.

3.3. HC dimension on a Nash real-analytic set. Independently of the above, one can show that an irreducible real-algebraic set of pure dimension has constant $\mathrm{HC}$ dimension. This is a consequence of the following proposition.

Proposition 3.5. Let $X$ be an irreducible $k$-dimensional real-algebraic set in $\mathbb{C}^{m}$. Then $\overline{\operatorname{Reg}_{k}(X)}$ is of constant $H C$ dimension. Moreover, there is a unique smallest pure-dimensional complex-algebraic set $Z$ in $\mathbb{C}^{m}$ such that $X \subset Z$ and $\operatorname{dim}_{\mathbb{C}} Z$ is equal to the constant $H C$ dimension of $\overline{\operatorname{Reg}_{k}(X)}$.

Proof. Set $Y:=\overline{\operatorname{Reg}_{k}(X)}$. Let $d:=\max \left\{\operatorname{dim}_{H C} Y_{p}: p \in Y\right\}$, and suppose that $\mathcal{S}^{d}(Y)$ is a proper subset of $Y$. Let $E_{1}, \ldots, E_{s}$ be the connected components of $\operatorname{Reg}_{k}(X)$. Then $Y=\bar{E}_{1} \cup \cdots \cup \bar{E}_{s}$. By Proposition 2.3, for each $j=1, \ldots, s$, there is a unique smallest irreducible complex-algebraic set $Z_{j}$ in $\mathbb{C}^{m}$ such that $\bar{E}_{j} \subset Z_{j}$ and $\operatorname{dim}_{\mathbb{C}} Z_{j}=\operatorname{dim}_{H C}\left(E_{j}\right)_{p}$ for all $p \in E_{j}$. By our hypothesis, there exists $j_{0}$ for which $\operatorname{dim}_{\mathbb{C}} Z_{j_{0}}<d$. Set

$$
\tilde{Z}:=\bigcup_{\operatorname{dim} Z_{j}<d} Z_{j}
$$

Then $\operatorname{dim}_{\mathbb{C}} \tilde{Z}<d$ and, hence, $Y \not \subset \tilde{Z}$. On the other hand, $\tilde{Z} \cap X \supset E_{j_{0}}$ and so $\operatorname{dim}(\tilde{Z} \cap Y)=\operatorname{dim} E_{j_{0}}=k$. Therefore, the real algebraic set $\tilde{Z} \cap X$ is of dimension 
$k$ and is a proper subset of $X$. But this is impossible, because $X$ is $k$ dimensional and irreducible. The contradiction proves that $\mathcal{S}^{d}(Y)=Y$.

For the final assertion of the proposition take $Z=\bigcup_{j=1}^{s} Z_{j}$, where the $Z_{j}$ are as above. Then $Z \cap X \supset Y$, hence $\operatorname{dim}(Z \cap X)=k$. Thus, by irreducibility of $X$, $Z \cap X=X$ and so $X \subset Z$. By the choice of $Z_{j}, Z$ is of pure (complex) dimension $d$, which is the constant $\mathrm{HC}$ dimension of $Y$.

Corollary 3.6. If $X$ is a pure-dimensional irreducible real-algebraic set in $\mathbb{C}^{m}$, then $X$ is of constant $H C$ dimension. Moreover, there is a unique smallest puredimensional complex algebraic set $Z$ in $\mathbb{C}^{m}$ such that $X \subset Z$ and $\operatorname{dim}_{\mathbb{C}} Z$ is equal to the constant $H C$ dimension of $X$.

Proposition 3.5 can be, in fact, generalized to the case of Nash real-analytic sets. This is interesting, because an anologous result is false in the transcendental case, as is shown by [4, Example 6.1].

Proposition 3.7. Let $R$ be a Nash real-analytic set in $\mathbb{C}^{m}$ (in the sense of [7]). If $R$ is irreducible (as a real-analytic set) and of dimension $k$, then $\overline{\operatorname{Reg}_{k}(R)}$ is of constant $H C$ dimension. Moreover, there is a unique smallest pure-dimensional complex-algebraic set $Z$ in $\mathbb{C}^{m}$ such that $R \subset Z$ and $\operatorname{dim}_{\mathbb{C}} Z$ is equal to the constant $H C$ dimension of $\overline{\operatorname{Reg}_{k}(R)}$.

Proof. Set $S:=\overline{\operatorname{Reg}_{k}(R)}$. Let $d:=\max \left\{\operatorname{dim}_{H C} S_{p}: p \in S\right\}$, and suppose that $\mathcal{S}^{d}(S)$ is a proper subset of $S$. Let $E_{1}, \ldots, E_{s}$ be the connected components of $\operatorname{Reg}_{k}(S)$. Then $S=\bar{E}_{1} \cup \cdots \cup \bar{E}_{s}$. As in the proof of Proposition 3.5, for each $j=1, \ldots, s$, there is a unique smallest irreducible complex-algebraic set $Z_{j}$ in $\mathbb{C}^{m}$ such that $\bar{E}_{j} \subset Z_{j}$ and $\operatorname{dim}_{\mathbb{C}} Z_{j}=\operatorname{dim}_{H C}\left(E_{j}\right)_{p}$ for all $p \in E_{j}$. By our hypothesis, there exists $j_{0}$ for which $\operatorname{dim}_{\mathbb{C}} Z_{j_{0}}<d$. Set

$$
\tilde{Z}:=\bigcup_{\operatorname{dim} Z_{j}<d} Z_{j}
$$

Then $\operatorname{dim}_{\mathbb{C}} \tilde{Z}<d$, and hence $S \not \subset \tilde{Z}$. Set

$$
\tilde{S}:=\bigcup_{\operatorname{dim} Z_{j}<d} \bar{E}_{j} .
$$

Let $\tilde{X}$ be the Zariski closure of $\tilde{S}$, and let $X$ be the Zariski closure of $R$. Then $\operatorname{dim} \tilde{X}=\operatorname{dim} \tilde{S}=k$ and $\operatorname{dim} X=\operatorname{dim} R=k$, by [7, $\S 2.8]$. Observe that $\tilde{X}$ is a proper subset of $X$. Indeed, $R \not \subset \tilde{X}$, because $S \not \subset \tilde{Z}$ and $\tilde{X} \subset \tilde{Z}$, by construction. On the other hand, $X$ is irreducible: For if $X=X_{1} \cup X_{2}$ with $X_{1}, X_{2}$ real-algebraic proper subsets of $X$, then $R=\left(R \cap X_{1}\right) \cup\left(R \cap X_{2}\right)$ would be a decomposition of $R$ into proper real-analytic subsets, unless $R \subset X_{1}$ or $R \subset X_{2}$ which would contradict the minimality of $X$. Hence, an irreducible real-algebraic set $X$ contains the real-algebraic $\tilde{X}$ as a proper subset and $\operatorname{dim} \tilde{X}=\operatorname{dim} X$, which is impossible. This proves that $\mathcal{S}^{d}(S)=S$.

The remainder of the proof is the same as that of Proposition 3.5

\section{Proof of Theorem 1.1}

Given the results of the preceeding section, it is now easy to adapt Shafikov's proof of [14, Theorem 1.3] to the Nash setting. We shall therefore restrict ourselves to highlighting just the key points of the proof. 
First, note that the graph $\Gamma_{f}$ is semialgebraic, of pure dimension $k:=\operatorname{dim} M$. Moreover, $\Gamma_{f}$ is $\mathcal{A R}$-irreducible by [9, Proposition 5.1] and $\mathcal{A} \mathcal{R}$-irreducibility of $M$. Let $\tilde{M}$ be the subset of $M$ on which $f$ is CR. Since $\operatorname{Reg}_{k} \Gamma_{f}$ is dense in $\Gamma_{f}$, there exists $p \in \tilde{M}$ such that $(p, f(p)) \in \operatorname{Reg}_{k} \Gamma_{f}$. By [9, Theorem 5.2] the map $f$ is analytic outside a set $S_{f}$ with $\operatorname{dim} S_{f} \leq \operatorname{dim} M-2$. Therefore, the point $p$ can actually be chosen so that $\Gamma_{f}$ near $(p, f(p))$ is the graph of a smooth map on an open neighbourhood $U^{p}$ of $p$ in $M$.

If the $\mathrm{CR}$ codimension of $M$ is zero, then $M$ is a complex-analytic manifold and $f$ is holomorphic on $U^{p}$. Then the germ $\left(\Gamma_{f}\right)_{(p, f(p))}$ is complex-analytic, and hence $\Gamma_{f}$ is an irreducible complex-algebraic set in $\mathbb{C}^{m} \times \mathbb{C}^{n}$, by Theorem 1.4. Let $\pi: \mathbb{C}^{m} \times \mathbb{C}^{n} \rightarrow \mathbb{C}^{m}$ and $\pi^{\prime}: \mathbb{C}^{m} \times \mathbb{C}^{n} \rightarrow \mathbb{C}^{n}$ be the projections. Then $\left.\pi\right|_{\Gamma_{f}}: \Gamma_{f} \rightarrow M$ is a bijective holomorphic mapping and, hence, a biholomorphism (see, e.g., 8, $\S 3.3$, Proposition 3]). It follows that $f=\left.\pi^{\prime}\right|_{\Gamma_{f}} \circ\left(\left.\pi\right|_{\Gamma_{f}}\right)^{-1}$ is holomorphic on the whole $M$. If, in turn, the CR dimension of $M$ is zero, then there is nothing to show because any function is CR on $M$.

Suppose then that $M$ is a CR manifold of type $(k, l)$ with both $k$ and $l$ positive. This part of the proof requires reduction to the case of a generic CR submanifold. It should be observed that, by the proof of [4, Proposition 1.4], a local embedding of $M$ into $\mathbb{C}^{k+l}$ (which makes $M$ generic) can be chosen semialgebraic. Therefore passing through that embedding does not affect the semialgebraicity of $M$ or $f$. The remainder of the proof follows exactly as in [14, with one major simplification: Namely, as a consequence of Theorem 1.3, in our case the graph $\Gamma_{f}$ has constant $\mathrm{HC}$ dimension $m$, and it is contained in an irreducible complex-algebraic subset $Z$ of $\mathbb{C}^{m} \times \mathbb{C}^{n}$ of dimension $m$ whose projection to $\mathbb{C}^{m}$ is generically finite over $M$. Therefore, one only needs to remove CR singularities of $f$ over the intersection of $M$ with a complex-algebraic subset $\Sigma$ of $\mathbb{C}^{m}$, defined as the closure of the projection of the algebraic-constructible set

$$
\operatorname{Sng} Z \cup\left\{z \in \operatorname{Reg} Z: \operatorname{rank} d_{z}\left(\left.\pi\right|_{Z}\right)<m\right\} .
$$

\section{REFERENCES}

[1] Janusz Adamus and Marcin Bilski, On Nash approximation of complex analytic sets in Runge domains, J. Math. Anal. Appl. 423 (2015), no. 1, 229-242, DOI 10.1016/j.jmaa.2014.09.070. MR 3273177

[2] Janusz Adamus and Serge Randriambololona, Tameness of holomorphic closure dimension in a semialgebraic set, Math. Ann. 355 (2013), no. 3, 985-1005, DOI 10.1007/s00208-0120808-y. MR.3020150

[3] Janusz Adamus, Serge Randriambololona, and Rasul Shafikov, Tameness of complex dimension in a real analytic set, Canad. J. Math. 65 (2013), no. 4, 721-739, DOI 10.4153/CJM2012-019-4. MR 3071076

[4] Janusz Adamus and Rasul Shafikov, On the holomorphic closure dimension of real analytic sets, Trans. Amer. Math. Soc. 363 (2011), no. 11, 5761-5772, DOI 10.1090/S0002-9947-201105276-1. MR2817408 (2012f:32010)

[5] M. Salah Baouendi, Peter Ebenfelt, and Linda Preiss Rothschild, Real submanifolds in complex space and their mappings, Princeton Mathematical Series, vol. 47, Princeton University Press, Princeton, NJ, 1999. MR.1668103 (2000b:32066)

[6] Edward Bierstone and Pierre D. Milman, Arc-analytic functions, Invent. Math. 101 (1990), no. 2, 411-424, DOI 10.1007/BF01231509. MR1062969(92a:32011)

[7] Jacek Bochnak, Michel Coste, and Marie-Françoise Roy, Real algebraic geometry, Ergebnisse der Mathematik und ihrer Grenzgebiete (3) [Results in Mathematics and Related Areas (3)], vol. 36, Springer-Verlag, Berlin, 1998. Translated from the 1987 French original; Revised by the authors. MR 1659509 (2000a:14067) 
[8] E. M. Chirka, Complex analytic sets, Mathematics and its Applications (Soviet Series), vol. 46, Kluwer Academic Publishers Group, Dordrecht, 1989. Translated from the Russian by R. A. M. Hoksbergen. MR:1111477 (92b:32016)

[9] Krzysztof Kurdyka, Ensembles semi-algébriques symétriques par arcs (French), Math. Ann. 282 (1988), no. 3, 445-462, DOI 10.1007/BF01460044. MR967023 (89j:14015)

[10] Krzysztof Kurdyka and Adam Parusiński, Arc-symmetric sets and arc-analytic mappings (English, with English and French summaries), Arc spaces and additive invariants in real algebraic and analytic geometry, Panor. Synthèses, vol. 24, Soc. Math. France, Paris, 2007, pp. 33-67. MR2409688

[11] Stanisław Łojasiewicz, Introduction to complex analytic geometry, Birkhäuser Verlag, Basel, 1991. Translated from the Polish by Maciej Klimek. MR 1131081 (92g:32002)

[12] Raghavan Narasimhan, Introduction to the theory of analytic spaces, Lecture Notes in Mathematics, No. 25, Springer-Verlag, Berlin-New York, 1966. MR0217337 (36 \#428)

[13] B. V. Shabat, Introduction to complex analysis. Part II, Functions of several variables, Translations of Mathematical Monographs, vol. 110, American Mathematical Society, Providence, RI, 1992. Translated from the third (1985) Russian edition by J. S. Joel. MR1192135 (93g:32001)

[14] Rasul Shafikov, Real analytic sets in complex spaces and CR maps, Math. Z. 256 (2007), no. 4, 757-767, DOI 10.1007/s00209-006-0100-5. MR2308889 (2008e:32008)

[15] Piotr Tworzewski, Intersections of analytic sets with linear subspaces, Ann. Scuola Norm. Sup. Pisa Cl. Sci. (4) 17 (1990), no. 2, 227-271. MR1076253 (91j:32008)

Department of Mathematics, The University of Western Ontario, London, Ontario, Canada N6A 5B7 - And - Institute of Mathematics, Polish Academy of Sciences, ul. ŚNIADECKICH 8, 00-956 WARsaW, Poland

E-mail address: jadamus@uwo.ca 